\title{
Crónica de internacional y su adaptación al entorno periodístico digital: Ios casos de El País y El Confidencial (España)
}

\section{Álvaro López-Martín 1 Bernardo Gómez-Calderón ${ }^{2}$}

Recibido: 30/09/2019

Aprobado por pares: 31/10/2019
Enviado a pares: 30/09/2019

Aceptado: 05/11/2019

DOI: 10.5294/pacla.2021.24.1.4

\section{Para citar este artículo / to reference this article / para citar este artigo}

López-Martín, A. y Gómez-Calderón, B. (2021). Crónica de internacional y su adaptación al entorno periodístico digital: Ios casos de El País y El Confidencial (España). Palabra Clave, 24(1), e2414. https://doi.org/10.5294/pacla.2021.24.1.4

\section{Resumen}

En los últimos dos decenios, la prensa ha experimentado una notable transformación, motivada, en gran medida, por la aparición de internet y su conversión en soporte informativo predominante, lo que ha influido decisivamente en la configuración de los géneros periodísticos clásicos. El objetivo de esta investigación consiste en identificar, a través del análisis de cien piezas publicadas durante 2018 en los diarios españoles El País y El Confidencial, cuáles son los rasgos que caracterizan la crónica internacional en el nuevo entorno virtual. Los resultados revelan que la adaptación online del género se ha traducido, sobre todo, en la incorporación de material audiovisual e hipertextos, que completan el repertorio de elementos tradicionalmente asociados a esta modalidad textual, como el background, 
o la anticipación de escenarios futuros. Esto nos lleva a concluir que la implantación del ciberperiodismo ha permitido a la crónica profundizar en sus cualidades informativas e interpretativas, convirtiéndola en un subgénero más valioso.

\section{Palabras clave (Fuente: tesauro de la Unesco)}

Periodismo; internet; prensa de información diaria; crónica; información internacional; medios de comunicación. 


\section{World Chronicle and its Adaptation to the Digital Journalistic Environment: The Cases of El País and El Confidencial (Spain)}

\section{Abstract}

In the last two decades, the press has undergone a remarkable transformation, motivated chiefly by the emergence of the Internet and its turning into the predominant information medium, which has decisively influenced the configuration of classic journalistic genres. By analyzing 100 pieces published during 2018 in the Spanish newspapers El País and El Confidencial, this research aims to identify the features that characterize world news in the novel virtual environment. The results reveal that the online adaptation of the genre has resulted in incorporating audiovisual material and hypertexts, completing the repertoire of elements traditionally associated with this textual modality, such as the background or the anticipation of future scenarios. In brief, the introduction of cyberjournalism has allowed world news to deepen its informative and interpretive traits, making it a more valuable subgenre.

\section{Keywords (Source: Unesco Thesaurus)}

Journalism; internet; newspaper press; chronicle; international information; mass media. 


\section{Crônica internacional e sua adaptação ao ambiente jornalístico digital: 0 caso de El País e de El Confidencial (Espanha)}

\section{Resumo}

Nas últimas duas décadas, a impressa experimentou uma notável transformação, motivada, em grande parte, pelo surgimento da internet e sua versão em suporte informativo predominante, o que vem influenciando decisivamente na configuração dos gêneros jornalísticos clássicos. O objetivo desta pesquisa consiste em identificar, por meio da análise de 100 peças publicadas durante 2018 nos jornais espanhóis El País e El Confidencial, quais os traços que caracterizam a crônica internacional no novo ambiente virtual. Os resultados revelam que a adaptação on-line do gênero tem se traduzido, principalmente, na incorporação de material audiovisual e hipertextos, que completam o repertório de elementos tradicionalmente associados com essa modalidade textual, como o background ou a antecipação de cenários. Isso nos leva a concluir que a implementação do ciberjornalismo tem permitido à crônica aprofundar em suas qualidades informacionais e interpretativas, convertendo-a em um subgênero mais valioso.

\section{Palavras-chave (Fonte: tesauro da Unesco)}

Jornalismo; internet; imprensa de informações diárias; crônica; informação internacional; meios de comunicação. 


\section{Introducción}

La transformación de la prensa en las últimas décadas, acelerada especialmente a causa del desarrollo de las ediciones online de los diarios, ha transformado de manera notable el modo en que los periodistas procesan la información de actualidad. Los géneros tradicionales han mutado para sobrevivir en los nuevos soportes surgidos en el entorno virtual, y lo han hecho con fortuna en la mayor parte de los casos, ya que han visto incrementado su potencial comunicativo.

Uno de esos géneros, de vitalidad contrastada especialmente en las áreas española y latinoamericana, es la crónica, muy frecuente para el relato cronológico de acontecimientos que requieren el testimonio in situ del informador. De entre las variedades textuales que la crónica cobija, la de internacional goza de especial rutilancia por lo exótico de su contenido para la audiencia. Los vínculos políticos y económicos cada vez más estrechos entre territorios geográficamente distantes, en esencia, la globalización, despiertan en la ciudadanía un interés lógico por las novedades del extranjero, lo cual lleva a los medios a otorgarles protagonismo en su agenda.

La crónica de internacional ha merecido hasta la fecha solo un interés moderado por parte de la academia, pese a venir practicándose desde la aparición de la prensa como fenómeno de masas y a exhibir un repertorio de cualidades bien definido, o quizá precisamente por ello. Si bien se localizan estudios que ponen el foco en la cobertura de acontecimientos concretos, sobre todo vinculados a conflictos bélicos (Lavín de las Heras y Römer, 2015; Tulloch, 2004), las investigaciones recientes en torno a la crónica internacional no abundan, a pesar de las mutaciones apreciables que ha experimentado el género con la implantación de la prensa digital, cuyos contenidos vienen determinados por factores como la multimedialidad, la interactividad y la hipertextualidad (Díaz-Noci, 2010). Creemos justificado, en consecuencia, abordar una aproximación a la crónica en el contexto del ciberperiodismo, con el propósito fundamental de determinar el uso que actualmente hacen los medios de ella, tomando como base la prensa española de referencia. 


\section{La crónica de internacional y su imperfecta indefinición}

El género de la crónica, encuadrado en el grupo de los textos interpretativos, supone, en palabras de Gil (2004, p. 26), la modalidad periodística "más adecuada" para relatar "a un determinado público lo que sucede en un lugar”. Quizá ahí radica una de las principales razones que explican la vigencia de este género en la prensa de habla hispana desde hace décadas.

Como apunta Rodríguez (2005, p. 168), “las crónicas periodísticas han gozado tradicionalmente de una alta consideración en la información”, sobre todo en España y en los países latinoamericanos, donde se ha convertido en "una modalidad [... ] de largo aliento" (Palau-Sampio, 2018, p. 195). Pese a su contrastada relevancia en la agenda mediática, la existencia de múltiples variedades del género y la multiplicidad de usos a que se destina hacen que no exista consenso a la hora de definir la crónica por parte de los investigadores que la han abordado, careciendo el género de cierta imprecisión ontológica (Moreno, 2007; Palau-Sampio, 2018). De manera predominante, "la crónica toma elementos de la noticia, del reportaje y del análisis”, distinguiéndose de estos dos últimos por la incorporación de elementos noticiosos, a lo que suma, ya como rasgo exclusivo, "una visión personal del autor” (Jordán, 2016, p. 37). Además, Moreno (2000) le confiere tres propiedades ineludibles: la limitación en el tiempo y en el espacio de los sucesos que cubre, la necesidad de que el informador adquiera la condición de testigo de los hechos y la inclusión de juicios personales en la narración de los acontecimientos.

Tomando el concepto de crónica en su más amplia acepción, Moreno (2000) distingue entre aquellos textos que se elaboran para "informar de todo cuanto suceda en un lugar concreto", elaborados por corresponsales o enviados especiales, y los que se hacen en atención a "la especialización que el cronista manifiesta en un determinado tema” (p. 177). De acuerdo con este criterio, la crónica de internacional se ubicaría en el primer grupo; pero no puede obviarse el acervo de conocimientos que la cobertura de la actualidad del extranjero exige al periodista, sin el cual sería imposible que desempeñara satisfactoriamente su labor. La dicotomía de 
Moreno, que, por otra parte, es tradicional en la periodística latina, resulta de este modo insuficiente para delimitar nuestro objeto de estudio.

Pero más allá de la hibridación que caracteriza al género, lo cierto es que la modalidad de internacional comparte con el resto de variedades de la crónica un factor, el del propósito o finalidad con el que es elaborada. En realidad, debe cumplir un cuádruple objetivo, ya que, como apunta Gil (2004), a la crónica se le exige “a) comunicación periódica por esencia $[\ldots]$; b) información por necesidad $[\ldots]$; c) orientación a los receptores [... ] y finalmente d) entretenimiento" (p. 30). En el caso de la variante internacional, el propósito de orientar a la audiencia es de mayor importancia, dada la complejidad de la información que se transmite y la necesidad de contextualización.

Ese redactor, el corresponsal, "representa una figura esencial en la tradición del periodismo del siglo XX” (León-Gross et al., 2018, p. 814). De hecho, para Galtung (2015, p. xxii), se necesitan corresponsales extranjeros en todo el mundo para trasladarnos lo que es realmente relevante.

Pero los cronistas de internacional atraviesan en estos momentos una situación delicada. La gran recesión de la segunda década del siglo XXI "ha provocado la precarización de la profesión de los informadores de manera global" (San Pablo, 2016, p. 9). Convertidos en el colectivo más vulnerable, los periodistas que informaban desde el extranjero han visto cómo por todo el mundo, y al margen de los medios a los que se adscribieran, sus puestos de trabajo (las corresponsalías) han ido desapareciendo mientras las empresas se ocupaban solo de la cuenta de resultados.

El estatus de los responsables de la información internacional se ha degradado inevitablemente. No obstante, todavía en época de bonanza, Haubrich (2005) ya llamaba la atención sobre la reducción del presupuesto que las grandes corporaciones mediáticas destinaban a sus corresponsales, apuntando a la irrupción de internet y al uso cada vez más frecuente de notas de las agencias de prensa como factores que impulsaban esta jibarización de la sección de internacional. 
También León-Gross et al. (2018) inciden en el pernicioso efecto que el uso generalizado de la red ha tenido para la figura del cronista clásico: "El desarrollo tecnológico y la aparición del denominado periodismo ciudadano han determinado que la información fluya por todo el planeta con una alta instantaneidad, propiciando que la figura del corresponsal haya llegado a ser cuestionada” (p. 814).

\section{La reinvención del periodismo en la era de internet: el caso español}

La irrupción de internet y de las redes sociales ha supuesto un hito revolucionario en la historia de la comunicación social. A consecuencia de su uso generalizado, el periodismo se ha visto arrastrado a un entorno online "que evoluciona de la mano del desarrollo de tecnologías $[. .$.$] y de cam-$ bios en los modelos de negocio en el sector de la información” (Peñafiel, 2016, p. 166).

En el caso de España, la primera publicación periodística disponible en internet data de 1994, se trataba de un artículo de El País firmado por Vicente Verdú. Meses más tarde aparecerían las ediciones web de $\mathrm{El} \mathrm{Pe}$ riódico de Catalunya y El Mundo, de ahí que dicho año sea considerado el punto de partida de la prensa digital en España (Díaz-Noci, 2005). En los Estados Unidos, la realidad era ya otra, más avanzada: en esa época, "entre los medios grandes y pequeños [...] contar con una edición electrónica se había convertido ya en un asunto de competencia y de prestigio" (Salaverría, 2005, p. 10), una tendencia que en pocos años llegaría también a los diarios españoles.

El periodismo digital ha traído aparejado un nuevo ecosistema mediático que implica una mutación tanto del ejercicio profesional como de los modelos de negocio de las empresas informativas, en las cuales se aprecia, además, un particular interés por poner en práctica iniciativas novedosas. En este sentido, la consolidación del entorno online ha ocasionado que "la presencia en internet no sea una recomendación, sino una obligación" (Cabrera et al., 2016, p. 43). No obstante, como sostiene Díaz-Noci (2010), 
el "gran reto" del sector en este nuevo contexto es lograr "la integración de la hipertextualidad, la multimedialidad y la interactividad" (p. 566), rasgos fundamentales de la comunicación ciberperiodística.

No son pocos los autores que ven en internet, antes que un problema, la vía para superar la crisis económica que afecta a los medios, ya que con la migración de la prensa a la web desaparecen los costes tradicionales de producción e impresión (Castañares y Mantini, 2015). Sin embargo, veinticinco años después del surgimiento de las primeras ediciones online, los grandes rotativos españoles aún se encuentran ensayando fórmulas para conseguir rentabilizar el producto periodístico digital, ya que los modelos de pago puestos en marcha hasta la fecha no han generado los beneficios que se esperaban. Primero, se apostó por el "gratis total", a lo que sucedieron las ediciones por suscripción, tras lo cual se pasó de nuevo a la oferta de contenidos en abierto, aunque con restricciones, etc.; todo en una carrera en la que pocos saben qué mecánica reportará finalmente dividendos y se convertirá en una opción estable. Como apunta Salaverría (2016), para las publicaciones en internet, la asignatura pendiente sigue siendo "hallar un modelo de negocio rentable y sostenible” (p. 206).

Del mismo modo, la implantación del paradigma digital ha supuesto el nacimiento de narrativas híbridas, cuyo propósito es hacer más atractiva la información para los lectores, y de nuevos géneros periodísticos, que, como apuntan Salaverría y Cores (2005), "son respuestas culturales a demandas sociales surgidas en un contexto histórico determinado. Su aparición, por tanto, no es gratuita: los géneros nacen siempre de la necesidad de satisfacer una función social" (p. 145). Paralelamente, los formatos tradicionales, como la crónica, se han visto abocados a la convergencia digital, la cual, en palabras de Serdali et al. (2016), la cual, según Serdali et al. (2016), debe entenderse como la realización de su potencial en un entorno informativo online (usualmente internet), en el cual los términos "género", "texto" y "pieza" serían reemplazados por "producto multimedia". Es en este entorno donde los moldes clásicos se ven sometidos a una profunda redefinición, y es donde se incardina este estudio. 


\section{Objetivos}

El objetivo fundamental de este trabajo estriba en identificar cuáles son los rasgos que caracterizan a la crónica en su modalidad de internacional, tal y como se cultiva en las versiones digitales de los diarios españoles de referencia. Ello debía permitir profundizar en el conocimiento de este subgénero, de larga y fructífera tradición en el periodismo latino, y determinar, en última instancia, las implicaciones que la adaptación al entorno web ha tenido para él.

Más allá de este propósito básico, y para una mayor concreción, se establecieron varios objetivos subsidiarios. En primer lugar, nos interesaba detectar las diferencias y similitudes que puedan apreciarse en el uso que de la crónica internacional hacen diarios de distinta naturaleza, híbridos o con referente papel y nativos digitales. Otro de los aspectos en los que se centraría el análisis sería en determinar las áreas geográficas a las que mayor atención se presta, ofreciendo un mapa de interés de los medios nacionales que revelara su criterio editorial implícito o manifiesto. En tercer lugar, se pretendía calibrar en qué medida las crónicas online han incorporado a su discurso los elementos característicos del ciberperiodismo, tales como el hipertexto o la documentación audiovisual. Y, por último, animaba nuestra investigación el propósito de contribuir, en la medida en que los resultados lo permitieran, a actualizar el concepto de crónica internacional de acuerdo con su práctica en los nuevos medios.

\section{Metodología}

El método de investigación aplicado en este trabajo, de vocación cuantitativa, ha sido el análisis de contenido. La elección de esta técnica como herramienta heurística obedeció al hecho de que ofrece una alta fiabilidad para el estudio exhaustivo de los mensajes periodísticos, tal y como advierten, entre otros, Igartua (2006), Krippendorff (1990) y Neuendorf (2002).

Se pone el foco sobre las crónicas de internacional publicadas en las versiones digitales de los diarios españoles de referencia. Dado que el universo de estudio era amplio, se optó por escoger dos cabeceras, El País y $E l$ Confidencial, de las cuales se seleccionó, a través de muestreo aleatorio sim- 
ple, 100 piezas (50 por medio) publicadas entre el 1 de enero y el 31 de diciembre de 2018, que constituirían las unidades de análisis.

La elección de las cabeceras respondió a criterios eminentemente cuantitativos, basados en las cifras de audiencia que ambas cosechan. En el ranking nacional, El País es el periódico en papel con edición online, o medio híbrido, con mayor número de lectores; según el medidor de referencia ComScore, 20394000 usuarios únicos accedieron durante marzo de 2019 a su web, mientras que $E l$ Confidencial hace años que constituye el medio nativo digital español con mejores datos de audiencia: en el citado mes, registró 13401000 usuarios únicos.

La ficha de análisis se estructuró en tres bloques, integrados a su vez por diversas variables:

1. Información de registro, para identificar la pieza en la matriz de datos de la investigación. Incluía los siguientes ítems:

a. Medio de publicación

b. Fecha

c. Autoría

2. Contenido. En este bloque, se contemplaron las variables relativas a la serie lingüística, lo que nos permitiría determinar el tratamiento periodístico que aplican las cabeceras seleccionadas a los hechos informativos internacionales.

a. Tema. Aquellos acontecimientos sobre los que se pone el foco en los textos analizados. Para un mejor estudio y clasificación, los distintos asuntos se categorizaron en trece bloques:

- Conflictos bélicos o diplomáticos

- Procesos electorales

- Desastres (accidentes humanos, naturales o medioambientales y atentados)

- Formación de gobiernos

- Relaciones internacionales/política exterior

- Encuentros o cumbres internacionales 
- Asuntos judiciales

- Relaciones comerciales

- Migración

- Protestas o manifestaciones

- Legislación

- Brexit

- - Generalidades

b. Área geográfica. Propuesta para identificar cuáles son las zonas con mayor peso en la cobertura internacional de la prensa española. Al respecto, se distinguieron ocho demarcaciones territoriales: África, América Latina, América del Norte, Extremo Oriente, Oriente Medio, Oriente Próximo, Unión Europea y resto de Europa.

c. Background. Uno de los rasgos característicos de la crónica es el suministro de información contextualizadora sobre los acontecimientos que aborda. Por ello, se atendió a los elementos narrativos y visuales destinados a dotar de sentido completo a las noticias, distinguiendo cuatro tipos:

- Textual

- Hipertextual, a través de la inclusión de enlaces que derivaran a contenidos que suministraran las claves diacrónicas de los hechos de actualidad

- Gráfico, basado en fotografías, vídeos, mapas e infografías

- Combinación de las anteriores

d. Previsiones. Nos ocupamos aquí de las referencias que se hicieran en los textos a posibles consecuencias o efectos de los acontecimientos narrados, otra de las propiedades que caracterizan a la crónica frente al resto de géneros informativos e interpretativos.

e. Recursos hipertextuales. Se pretendía explorar con qué finalidad se recurre a hiperenlaces de carácter textual en las crónicas de internacional. Además de determinar su uso, interesaba registrar la frecuencia de utilización y la naturaleza de los materiales asociados a las piezas de la muestra. 
3. Elementos gráficos. Se incluyeron las variables relativas a los elementos paralingüísticos e icónicos (fotografías, vídeos, infografías, mapas, etc.) de las crónicas, figuraran incrustados en ellas o accesibles a través de enlaces hipermediales.

\section{Resultados}

\section{Temática}

Los resultados del análisis indican la existencia de cuatro bloques temáticos destacados tanto en El País como en El Confidencial: política exterior en sentido amplio, procesos electorales de distinta envergadura, negociaciones encaminadas a la formación de gobiernos $y$, como único suceso singularizado de la escala, el brexit, cuestión omnipresente desde 2016 en el debate público europeo. Los cuatro ámbitos señalados destacan por su transversalidad, esto es, por el hecho de que, con muy pocas excepciones, implican a más de un Estado, lo cual incrementa lógicamente el interés periodístico que suscitan.

De todos los acontecimientos que registran una incidencia elevada, son los comicios los que mayor cobertura reciben, protagonizando el $16 \%$ de las crónicas analizadas en El País y el $10 \%$ de las de El Confidencial, con preferencia por las elecciones de carácter presidencial. El año 2018 vino marcado por cambios en la jefatura del Estado de algunos de los países latinoamericanos de mayor peso en la agenda de los medios españoles, caso de Colombia o Brasil, además de por la renovación de la Presidencia de Nicolás Maduro en Venezuela; en todos los casos, y aunque por razones distintas, el interés informativo era elevado, de ahí que el número de piezas asociadas a ellos destaque en los dos diarios analizados.

Frente a esto, asuntos como los procesos judiciales internacionales, las relaciones comerciales entre países y territorios, las elecciones de carácter legislativo, los encuentros entre líderes políticos y, sorprendentemente, las migraciones y los comicios al Parlamento Europeo, tienen una presencia reducida en las piezas de la muestra (tabla 1). 


\section{Tabla 1. Temas de las crónicas analizadas}

\begin{tabular}{|c|c|c|}
\hline Tema & El País (\%) & El Confidencial (\%) \\
\hline Brexit & 8 & 14 \\
\hline Conflicto bélico o diplomático & 4 & 6 \\
\hline \multicolumn{3}{|l|}{ Desastres } \\
\hline Accidentes humanos & 4 & 6 \\
\hline Atentados & 6 & 2 \\
\hline Catástrofes naturales & 2 & - \\
\hline \multicolumn{3}{|l|}{ Elecciones } \\
\hline Consultas ciudadanas & 2 & - \\
\hline Al Parlamento Europeo & 2 & 2 \\
\hline Legislativas & 2 & - \\
\hline Presidenciales & 10 & 8 \\
\hline Encuentros o cumbres & 4 & 4 \\
\hline Formación de gobiernos & 10 & 8 \\
\hline Generalidades & 8 & 6 \\
\hline Procesos judiciales & 4 & 2 \\
\hline Legislación internacional & - & 12 \\
\hline Migración & 2 & 6 \\
\hline Protestas o manifestaciones & 8 & 2 \\
\hline Relaciones comerciales & 6 & 2 \\
\hline Relaciones internacionales & 18 & 20 \\
\hline Total & 100 & 100 \\
\hline
\end{tabular}

Fuente: elaboración propia.

\section{Área geográfica}

En materia geográfica, se constata que los países miembros de la Unión Europea (UE) son los que generan el mayor volumen de información internacional publicada por los medios analizados. Los asuntos procedentes de esta región centran el 41,07 \% de las crónicas de El Confidencial y el $36 \%$ de las de El País. Más concretamente, en ambos casos, la mayoría de las piezas abordan hechos acaecidos en Francia, Alemania, Italia y el Reino Unido, los cuatro países con mayor peso demográfico y económico de la UE.

Asimismo, como se recoge en la tabla 2, un porcentaje notable de crónicas aborda la actualidad de América Latina, territorio con el que Es- 
paña mantiene sólidos vínculos culturales, económicos y políticos, entre ellos el más evidente el idiomático. Mayor es la incidencia de esta área en la cobertura del diario del grupo Prisa que en la cabecera dirigida por $\mathrm{Na}$ cho Cardero (22\% frente a 14,29\%). En ello incide, probablemente y más allá de razones de índole periodística, el hecho de que El País ofrece online una edición regional para Brasil y cuenta con dos delegaciones en la zona (Ciudad de México y São Paulo), además de disponer de una corresponsalía en Buenos Aires.

Trasladando el foco a Asia, su presencia informativa es discreta salvo en lo referido a Extremo Oriente, que genera el 8,92 y el $8 \%$ de los textos analizados de El Confidencial y El País, respectivamente, siendo China y Corea del Norte los Estados que de modo más habitual aparecen en el análisis. Mientras tanto, cuatro países de Oriente Próximo figuran también en la agenda internacional de las cabeceras de la muestra: Arabia Saudí, Siria, Líbano e Israel, tradicionales focos noticiosos que a lo largo de 2018 mantuvieron el interés de los medios merced a los conflictos en que se encuentran inmersos. En el caso de África, no tiene presencia en El País y sí en El Confidencial, aunque su incidencia no alcanza cifras relevantes (5,36\%).

Tabla 2. Áreas geográficas generadoras de información

\begin{tabular}{|c|c|c|}
\hline Territorio & El País (\%) & El Confidencial (\%) \\
\hline África & - & 5,36 \\
\hline América del Norte & 14 & 14,29 \\
\hline América Latina & 22 & 14,29 \\
\hline Extremo Oriente & 8 & 8,92 \\
\hline Oriente Medio & 2 & - \\
\hline Oriente Próximo & 4 & 10,71 \\
\hline Resto de Europa & 14 & 41,07 \\
\hline Unión Europea & 36 & $\mathbf{1 0 0}$ \\
\hline Total & $\mathbf{1 0 0}$ & \\
\hline
\end{tabular}

Fuente: elaboración propia. 


\section{Antecedentes o background}

Si hay un subgénero que precisa de información contextualizadora, ese es el de la crónica en su modalidad internacional. El repertorio de datos referidos a los antecedentes de un hecho que el corresponsal despliegue en sus textos puede condicionar de modo decisivo la comprensión de los acontecimientos, habitualmente remotos, cuando no exóticos, que está trasladando a la audiencia. Como se muestra en la figura 1, la mayor parte de las piezas de los dos medios analizados ofrece background, en especial las de la cabecera de Prisa (90\%, 45 piezas). Levemente inferior es el dato de El Confidencial, en el que se contextualiza el 78 \% de las informaciones (39 unidades).

\section{Figura 1. Porcentaje de crónicas en las que se contextualiza.}

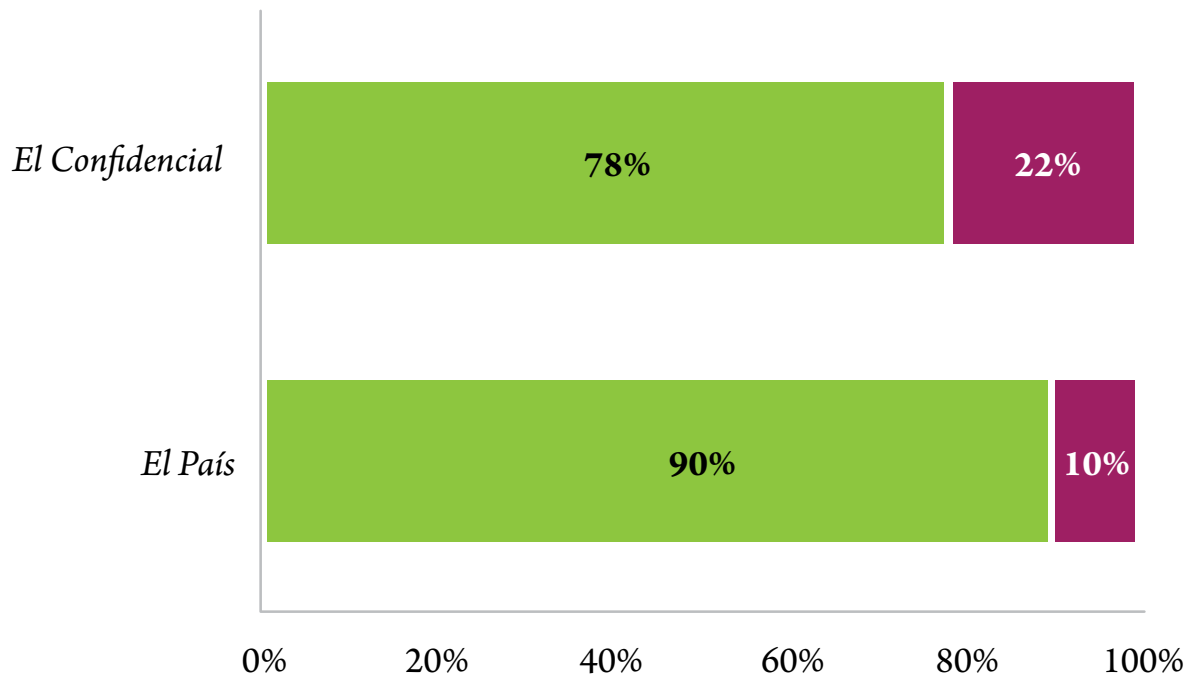

Sí aporta datos de antecedentes $\quad$ No contextualiza la información Fuente: elaboración propia.

El modo en que un cronista puede contextualizar los hechos que aborda ha variado desde la aparición del periodismo digital, aquí radica uno de los factores que más claramente diferencian a la prensa online de la de papel por lo que a la configuración de nuestro género se refiere. En la tabla 3, se recogen las diferentes herramientas que sirven de vehículo al background 
según los resultados del análisis. En El País, la inclusión de elementos textuales e hipertextuales en la propia pieza es la vía principal de suministro de antecedentes. La combinación de ambos métodos se registra en 21 de las crónicas de ese medio, lo que representa un 46,67 \%. Esta solución dual desempeña también un papel destacado en El Confidencial, donde se contabilizan 11 unidades $(28,2 \%)$ que aúnan elementos textuales e hipertextuales con el cometido de situar los acontecimientos.

Asimismo, la inclusión de background en el propio cuerpo de la crónica, sin enlaces, es otro método frecuentemente empleado por los dos medios objeto de estudio: en El Confidencial aportan antecedentes mediante este procedimiento el 41,03\% de los textos, frente al 35,56\% del diario de Prisa. En menor medida, se localizan otras fórmulas, como el suministro de elementos gráficos, aunque el uso que se hace de estos tiene siempre una incidencia modesta.

\section{Tabla 3. Herramienta empleada para la contextualización}

\begin{tabular}{|l|c|c|}
\hline \multicolumn{1}{|c|}{ Tipología } & El País (\%) & El Confidencial (\%) \\
\hline Textual & 35,56 & 41,03 \\
\hline Hipertextual & 4,44 & 15,38 \\
\hline Gráfico & 2,22 & - \\
\hline Textual + hipertextual & 46,67 & 28,20 \\
\hline Textual + gráfico & 4,44 & 2,56 \\
\hline Textual + hipertextual + gráfico & 6,67 & 12,83 \\
\hline Total & $\mathbf{1 0 0}$ & $\mathbf{1 0 0}$ \\
\hline
\end{tabular}

Fuente: elaboración propia.

\section{Previsiones}

En tanto que la contextualización del hecho informativo se ha demostrado una práctica habitual en la confección de las crónicas de internacional, otro rasgo característico del género, la anticipación de escenarios futuros que pu- 
dieran derivarse de los acontecimientos actuales, no ofrece en el análisis un patrón definido, ya que tanto en El País como en El Confidencial la proporción de textos que incluyen previsiones apenas supera el $50 \%$ de la muestra.

En términos cualitativos, los datos aportados sobre el posible desarrollo de los hechos noticiosos resultan, con frecuencia, imprecisos y vagos, limitándose en la mayoría de los casos a avanzar cuestiones de agenda, esto es, sucesos previstos, como la reunión de mandatarios o la celebración de efemérides, en los que la capacidad interpretativa del cronista, que es lo que aporta mayor valor a sus textos, no entra en juego, obviándose de manera muy habitual las implicaciones económicas y, sobre todo, sociales que puedan tener los acontecimientos relatados (figura 2).

\section{Figura 2. Porcentaje de crónicas en las que se aportan previsiones.}

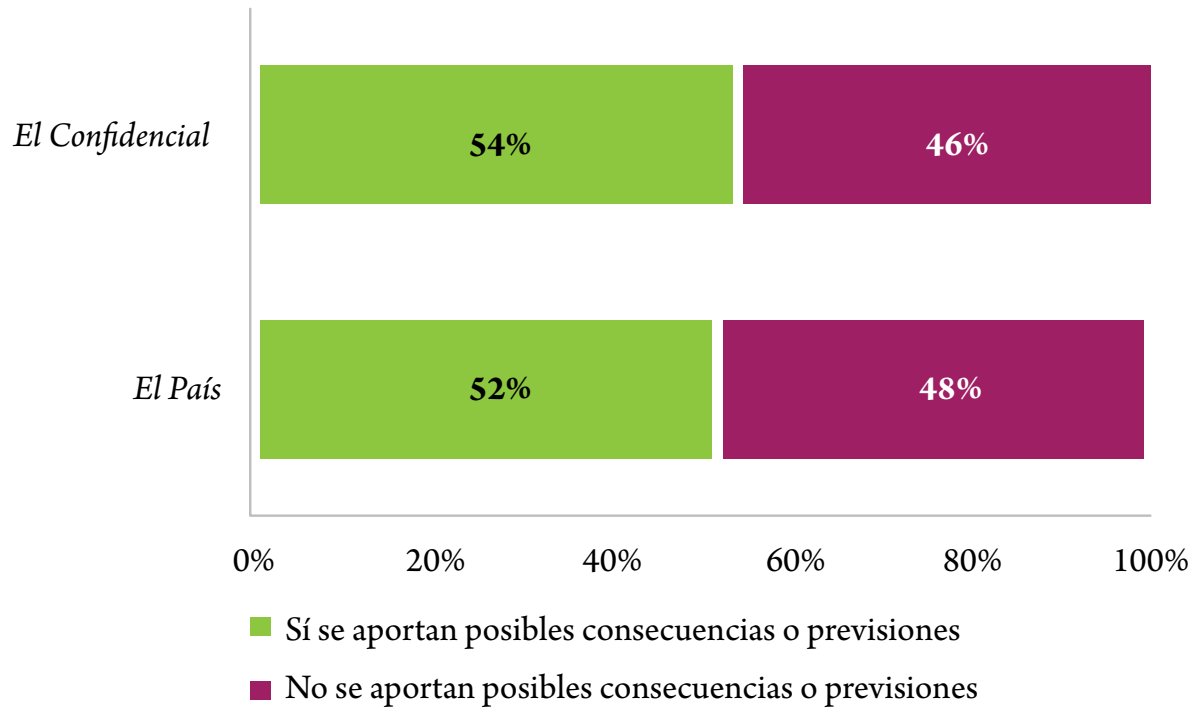

Fuente: elaboración propia.

\section{Hipertextualidad}

Como se ha apuntado ya, la hipertextualidad es uno de los rasgos definitorios del ciberperiodismo; históricamente es el primer recurso que se incorporó a los textos digitales y marcó la diferencia respecto de las versiones 
en papel. En la tabla 4, se refleja la inclusión de hipertextos en las crónicas analizadas. Si bien el dato del diario de Prisa es levemente superior al registrado por El Confidencial (6,68 ocurrencias por pieza frente a 6,02), en ambos casos se puede considerar muy similar el empleo de hipertextos, $y$ no solo en términos cuantitativos, sino que también la naturaleza del material enlazado suele ser la misma.

Lo habitual es que se utilicen para redirigir a noticias del mismo medio publicadas con anterioridad: así ocurre en 285 casos en El País (85,33\%) y en 257 en El Confidencial (85,39\%). Las otras modalidades más empleadas, aunque con una frecuencia de uso considerablemente menor, sirven para conducir al lector a informaciones aparecidas en otros canales o en páginas oficiales de instituciones, administraciones y personalidades de la esfera pública. En el primer caso, el objeto preferente de los hipertextos son medios de comunicación de los países donde suceden los acontecimientos, lo cual proporciona a aquellos lectores con la suficiente inquietud intelectual un saludable punto de vista alternativo que puede contrarrestar el tan denostado etnocentrismo del que con frecuencia se acusa a las crónicas publicadas por la prensa occidental. En muy raras ocasiones, los enlaces redirigen a otros medios españoles, no en vano competencia de El País y El Confidencial en la pugna por la captación de usuarios.

También vinculan, aunque en menor medida, los hiperenlaces a redes sociales, principalmente Twitter, de políticos, instituciones o testigos de los acontecimientos. En este último caso, los internautas se convierten en prosumidores y se posicionan, cada vez con más frecuencia, como fuente de la que se nutren los propios medios de comunicación. Por último, la discreta utilización de hipertextos enlazados a documentos completos vinculados al tema principal de la crónica (El Confidencial, 0,99\% de casos; El País, 2,06\%) evidencia una preferencia clara de las cabeceras analizadas por ofrecer a sus lectores la información sintetizada y jerarquizada, en lugar de material en bruto en el que orientarse puede ser difícil: la función de mediación de la prensa se impone aquí a las posibilidades de difusión masiva de datos propia del entorno virtual. 


\section{Tabla 4. Empleo de hipertextos en las crónicas*}

\begin{tabular}{|l|c|c|}
\hline Destino & El País & El Confidencial \\
\hline Noticias del mismo medio & $285(85,33 \%)$ & $257(85,39 \%)$ \\
\hline Otro medio & $21(6,28 \%)$ & $14(4,65 \%)$ \\
\hline Web oficial de instituciones o personalidades & $15(4,5 \%)$ & $12(3,99 \%)$ \\
\hline Documentos & $2(0,6 \%)$ & $3(0,99 \%)$ \\
\hline Twitter & $9(2,69 \%)$ & $15(4,98 \%)$ \\
\hline Facebook & $1(0,3 \%)$ & - \\
\hline YouTube & $1(0,3 \%)$ & - \\
\hline Total & $\mathbf{3 3 4 ( 1 0 0 \% )}$ & $\mathbf{3 0 1}(\mathbf{1 0 0} \%)$ \\
\hline Promedio hipertextos/pieza: & 6,68 & 6,02 \\
\hline
\end{tabular}

* Se indica el número de ocurrencias y, entre paréntesis, la proporción respecto del total de la muestra.

Fuente: elaboración propia.

\section{Elementos visuales}

La multimedialidad es otro de los rasgos que singularizan al producto ciberperiodístico. Los diarios tradicionales han ido incorporando de manera paulatina un repertorio amplio de recursos gráficos y audiovisuales a los textos de sus ediciones digitales, recursos que en la crónica internacional proporcionan, con frecuencia, datos o perspectivas muy útiles para la contextualización de los hechos narrados.

Como se recoge en la tabla 5, las fotografías y los vídeos son las dos herramientas visuales más empleadas en la información del extranjero. Mientras que en El País se contabilizan 54 imágenes, lo que supone una media de 1,08 por pieza, El Confidencial eleva el registro hasta las 105 en una muestra de textos de idéntica envergadura: su apuesta por el elemento gráfico se antoja mucho más decidida.

Por su parte, el otro recurso destacado, el vídeo, tiene un uso menor que la fotografía en El País y significativamente más reducido en El Confidencial, ya que presentan 39 y 11 piezas audiovisuales, respectivamente, en el total de unidades del análisis. Esto supone el uso de 0,78 elementos por texto en el diario de Prisa y 0,22 en la cabecera digital. Además de 
los datos relativos a la inclusión de estos recursos, su duración también apuntala la consideración de que El País dedica mayor atención a su producción y difusión.

Finalmente, los gráficos convencionales y los mapas presentan una incidencia limitada. Si bien en $\mathrm{El}$ Confidencial la utilización de ambos elementos es similar y, casi, insignificante, en El País se hace un uso algo más pronunciado de ellos, especialmente de los gráficos, de los que se contabilizan nueve, distribuidos en cinco crónicas.

\section{Tabla 5. Empleo de elementos visuales}

\begin{tabular}{|c|c|c|}
\hline Elemento & El País & El Confidencial \\
\hline Fotografías & & $\mathbf{1 0 5}$ \\
\hline Total & $\mathbf{5 4}$ & 2,1 fotografía/pieza \\
\hline Promedio inclusión & 1,08 fotografía/pieza & $\mathbf{1 1}$ \\
\hline Vídeos & & 0,22 vídeo/pieza \\
\hline Total & $\mathbf{3 9}$ & 1 ' 11" \\
\hline Promedio inclusión & 0,78 vídeo/pieza & $\mathbf{2}$ \\
\hline Promedio duración & 1 ' 38 ” & 0,04 gráfico/pieza \\
\hline Gráficos & & $\mathbf{2}$ \\
\hline Total & $\mathbf{9}$ & 0,04 mapa/pieza \\
\hline Promedio inclusión & 0,18 gráfico/pieza & \\
\hline Mapas & & $\mathbf{4}$ \\
\hline Total & 0,08 mapa/pieza & \\
\hline Promedio & & \\
\hline
\end{tabular}

Fuente: elaboración propia.

\section{Conclusiones}

La crónica en su modalidad de internacional sigue teniendo una notable vigencia en la prensa española de referencia, aunque no por ello ha dejado de experimentar las mutaciones a que se ha visto sometido el periodismo a causa de la implantación del paradigma digital, haciendo efectivas en diverso grado las potencialidades que ofrecen los nuevos formatos.

En relación con los objetivos que nos planteábamos al comienzo de la investigación, y atendiendo al primero, además de la propia naturaleza, 
híbrida o nativa digital, de cada diario analizado, una de las divergencias que se ha evidenciado en la cobertura internacional de El Confidencial y El País radica en el empleo de los elementos visuales. Aunque la cabecera de Prisa hace un uso moderado de la fotografía, en sus crónicas tiene una notable incidencia la inclusión de vídeos y, en menor medida, de gráficos y mapas; en todos estos terrenos, El Confidencial se muestra más parco. Frente a ello, en aspectos como los temas abordados o el uso de hipertextos, enlazados principalmente a noticias del propio medio, ambos rotativos arrojan registros muy similares. Hay, por tanto, y pese a la línea editorial diferenciada, convergencia de agendas en el plano internacional y estrategias periodístico-empresariales muy próximas.

Otro de los aspectos que ha aflorado en el análisis, cuya identificación se perseguía en el segundo objetivo, es el de las áreas geográficas sobre las que pivota la cobertura internacional de los diarios seleccionados. Tanto en El País como en El Confidencial, los tres territorios predominantes son la UE, América Latina y América del Norte, estos dos últimos con presencia destacada aunque menos regular. Continentes y regiones como África y Oriente Medio apenas ofrecen resultados, lo cual confirma que la atención que se presta a los acontecimientos del extranjero en los medios viene condicionada por los intereses nacionales, sean estos de naturaleza política, económica o cultural; en España, dichos intereses son innegables en relación con sus socios de la UE, con los Estados Unidos, principalmente en materia de defensa, y con toda América Latina, de ahí los resultados del análisis. La proximidad física, que en esta ocasión vendría representada por el Magreb, resultaría menos determinante en la confección de la agenda internacional de la prensa.

En cuanto a los rasgos ciberperiodísticos incorporados por la crónica (tercer objetivo), son los materiales audiovisuales y, sobre todo, los hipertextos, ambos muy abundantes en la muestra, los que en mayor medida se han asociado al género gracias a la convergencia digital, y marcado la diferencia entre la cobertura realizada en papel y la que se lleva a cabo en las ediciones online. Muchos de los vínculos externos, además, apuntalan la tarea de contextualización propia de la crónica, y ofrecen contenidos suple- 
mentarios, y lo que resulta más enriquecedor, acceso a las fuentes primarias de los acontecimientos.

Por último, y en lo que respecta a la definición de nuestro género en el periodismo digital (cuarto objetivo), el análisis ha puesto de manifiesto que la crónica internacional, pese a mantener el letimotiv tradicional de relato personalizado de acontecimientos lejanos, ha adquirido valor añadido al convertirse en producto online por medio del suministro abundante de material audiovisual y la incorporación de recursos externos que proporcionan antecedentes y claves de los acontecimientos, desterrando de este modo la unilateralidad de la mirada informativa e incrementando la pluralidad del discurso.

No se nos ocultan las limitaciones de nuestro estudio, toda vez que el corpus del análisis se ha ceñido solo a dos cabeceras. Pero, aun así, podemos concluir que, en el contexto ciberperiodístico, la crónica no solo no ha perdido sus rasgos definitorios, sino que los ha depurado. Las funciones interpretativas y explicativas que tradicionalmente le han estado encomendadas se han beneficiado de las posibilidades que ofrece el entorno virtual: donde antes una única perspectiva, la del cronista, marcaba el sentido de los acontecimientos, hay ahora una multiplicidad de voces que permite a la audiencia alcanzar un entendimiento de los hechos mucho más aquilatado, de mayor hondura y mejor construido. La crónica internacional, como otros géneros del periodismo clásico, ha ganado con la reconversión tecnológica; queda ahora que ganen también, y enlazamos aquí con el primer bloque de nuestro trabajo, quienes la hacen posible, los corresponsales, para los que la diversificación de medios no siempre se traduce en una mejora de las condiciones laborales.

\section{Referencias}

Cabrera Méndez, M., Lloret Romero, N. y Díez Somavilla, R. (2016). La importancia de la identidad digital del periodista. En H. M. Sánchez Gonzales (ed.), Nuevos retos para el periodista: Innovación, creación y emprendimiento (pp. 44-63). Tirant lo Blanc. 
Castañares, W. C. y Mantini, M. (2015). La transformación de las prácticas y rutinas profesionales en el periodismo contemporáneo: estudio de caso. Perspectivas de la Comunicación, 8(1), 115-130. http:// publicacionescienciassociales.ufro.cl/index.php/perspectivas/ article/view/499

Díaz-Noci, J. (2005). Historia de los cibermedios en España. En R. Salaverría (coord.), Cibermedios: El impacto de internet en los medios de comunicación en España (pp. 21-38). Comunicación Social.

Díaz-Noci, J. (2010). Medios de comunicación en internet: Algunas tendencias. El Profesional de la Información, 19(6), 561-567. https:// doi.org/10.3145/epi.2010.nov.01

Galtung, J. (2015). Foreing corresponde 50 years later. En G. Trezis (ed.), Mapping foreign correspondence in Europe (pp.xix-xxiii). Routledge. https://transnational.live/2020/03/03/foreign-correspondence-50-years-later/

Gil, J. C. (2004). La crónica periodística: Evolución, desarrollo y nueva perspectiva. Viaje desde la historia al periodismo interpretativo. Global Media Journal Edición Iberoamericana, 1(1), 26-39. https:// idus.us.es/handle/11441/24803

Haubrich, W. (2005). Corresponsales en España: Tres preguntas sobre la profesión y los medios. En P. Farias Batlle (dir.), Informe anual de la profesión periodística 2005 (pp. 61-67). Asociación de la Prensa de Madrid. https://www.apmadrid.es/publicaciones/informeanual-de-la-profesion/

Igartua, J. J. (2006). Métodos cuantitativos de investigación en comunicación. Bosch.

Jordán, D. (2016). La crónica, el género literario del periodismo que merece sobrevivir. INNOVA Research Journal, 1(12), 35-43. https:// doi.org/10.33890/innova.v1.n12.2016.138 
Krippendorff, K. (1990). Metodología del análisis de contenido: Teoría y práctica. Paidós.

Lavín de las Heras, E. y Römer Pieretti, M. (2015). Efectos de internet y las redes sociales en los corresponsales de guerra españoles. Icono 14, 13(2), 192-213. https://doi.org/10.7195/ri14.v13i2.783

León-Gross, T. A., Rivera-Hernández, A. R. y Redondo-Escudero, M. (2018). Los corresponsales internacionales en España ante el Gobierno y las fuentes: Evaluación de problemas mediante proceso de análisis jerárquico (PAJ).El Profesional de la Información, 27(4), 813-821. https://doi.org/10.3145/epi.2018.jul.10

Moreno, P. (2000). Los géneros periodísticos informativos en la actualidad internacional. Ámbitos, 5, 169-190. https://idus.us.es/handle/11441/67346

Moreno, P. (2007). Opinión y géneros en el periodismo electrónico: Redacciónyescritura.Ámbitos, 16, 123-149.https://idus.us.es/bitstream/ handle $/ 11441 / 12712 /$ file_1.pdf?sequence $=1$ \&isAllowed $=y$

Neuendorf, K. A. (2002). The content analysis guidebook. Sage.

Palau-Sampio, D. (2018). Las identidades de la crónica: Hibridez, polisemia y ecos históricos en un género entre la literatura y el periodismo. Palabra Clave, 21(1), 191-2018. https://doi.org/10.5294/ pacla.2018.21.1.9

Peñafiel, C. (2016). Reinvención del periodismo en el ecosistema digital y narrativas transmedia. AdComunica, 12, 163-182. https://doi. org/10.6035/2174-0992.2016.12

Rodríguez, C. (2005). Las crónicas: Algunas ideas sobre la credibilidad en el periodismo interpretativo. Estudios sobre el Mensaje Periodístico, 11, 167-180. https://revistas.ucm.es/index.php/ESMP/article/ view/ESMP0505110167A 
Salaverría, R. (coord.) (2005). Cibermedios: El impacto de internet en los medios de comunicación en España. Comunicación Social.

Salaverría, R. (coord.) (2016). Ciberperiodismo en Iberoamérica. Fundación Telefónica. https://dadun.unav.edu/handle/10171/42336

Salaverría, R. (2019). Digital journalism: 25 years of research. Review article. El Profesional de la Información, 28(1), e280101. https://doi. org/10.3145/epi.2019.ene.01

Salaverría, R. y Cores, R. (2005). Géneros periodísticos en los cibermedios hispanos. En R. Salaverría (coord.), Cibermedios: El impacto de internet en los medios de comunicación en España (pp. 145-185). Comunicación Social.

San Pablo, P. (2016). La información internacional 3.0: Las nuevas relaciones entre periodistas, redes sociales y ONG. En P. Moreno Espinosa (coord.), Comunicación, ciberperiodismo y nuevos formatos multimedia interactivos (pp. 8-31). Egregius.

Serdali, B. K., Ashirbekova, G. S., Orazbekuly, K. y Abiev, B. M. (2016). Genres of modern mass media. International Electronic Journal of Mathematics Education, 11(5), 1075-1085. https://www.iejme. com/article/genres-of-modern-mass-media

Tulloch, C. D. (2004). Corresponsales en el extranjero: Mito y realidad. Eunsa. 